Heat Transfer Research 46(2) (2015)

\title{
PREFACE: HEAT AND MASS TRANSFER IN SORPTION COOLING SYSTEMS
}

The collection of papers presented in this special issue mainly focus on the technology of the sorption refrigeration and heat pump (SRHP).

Refrigeration and heat pump (RHP) is a technology closely related with the people's living worldwide. The conventional RHP is driven by electricity; the common refrigerants it utilized are generally not environmentally benign (with significant ODP or GWP).

The SRHP is powered by the low-grade thermal heat, such as solar thermal energy and waste heat from industries or power systems. It has three advantages if compared with the conventional RHP. Firstly, it will save the energy of the electricity because it is driven by the low-grade heat. Secondly, it uses the green refrigerants (natural refrigerants like water, ammonia, etc.), thus it will not cause the ozone depletion problems. Thirdly, it will help in protecting the environment as some part of waste heat is used to produce useful refrigeration.

The drawbacks of the SRHP are the low COP (coefficient of performance) and low SCP (mainly for the solid adsorption system, and it is specific cooling power per kilogram adsorbents). Generally the COP of an advanced cycle of a sorption refrigeration system is lower than 0.6 , and the SCP of a solid adsorption refrigeration system with the technology of intensification of heat and mass transfer is lower than $350 \mathrm{~W} / \mathrm{kg}$. Both low COP and SCP will lead to a bulk system, which is the most critical problem for the promotion of the technology to commercialization.

To improve the COP and SCP of SRHP systems, the advanced cycles, intensification of heat and mass transfer performance, and new types of efficient adsorbents need to be investigated extensively. We are glad to say that all these topics are involved in this special issue. The systems driven by different types of low-grade heat, including solar energy and waste heat, are analyzed in this issue. Different types of advanced cycles, new technologies for the intensification of heat and mass transfer performances, and new types of efficient adsorbents are researched intensively.

We are glad to express our sincere thanks to all the invited authors; your contributions have brought a unique special issue for the technology development for SRHP. Our thanks also include the editorial committee of HTR - they gave us a very good chance for presenting the new developments in SRHP. Nowadays the fast speed of energy expenditure and the serious environment pollution are two tremendous prob-

1064-2285/15/\$35.00 @ 2015 by Begell House, Inc. 
lems worldwide. As a type of energy saving and environmental benign technology, the SRHP should be given an increasingly greater attention and get a fast development. It is hoped that this issue will give the readers an overall understanding of the latest technologies and can speed up further development of the SRHP.

\section{Guest Editors:}

Prof. Dr. Ruzhu Wang

Institute of Refrigeration \& Cryogenics

School of Mechanical Engineering

Shanghai Jiao Tong University, China

\section{Prof. Dr. Liwei Wang}

Institute of Refrigeration \& Cryogenics

School of Mechanical Engineering

Shanghai Jiao Tong University, China 